\title{
Perspectives of Mobile Cloud Computing: Architecture, Applications and Issues
}

\author{
Rupinder Pal Kaur \\ Assistant Professor, \\ Guru Nanak College for Girls, \\ Sri Muktsar Sahib, Punjab
}

\author{
Amanpreet Kaur \\ Assistant Professor \\ Guru Nanak College for Girls, \\ Sri Muktsar Sahib, Punjab
}

\begin{abstract}
With advancement of technology and rapid growth of mobile applications, Mobile Cloud Computing was introduced in mobile platform to serve needs of users. Mobile cloud addresses the problem of managing data while on moving. Mobile cloud helps in processing data outside the device which provide a great help to manage data securely. In this paper we have discussed about architecture both traditional and open mobster, some issues related to mobile cloud .we have also gone through applications and advantages that can be served through MCC.
\end{abstract}

Keywords: cloud computing, mobile cloud computing, open mobster, mobile cloud services.

\section{INTRODUCTION}

\section{Motivation}

Today the world is of mobile devices. Every single person carries a mobile device with him either its Smartphone, tablet pc etc. Mobile devices become a part of human life. With advancement of technology everyone need information at fingers. According to Bill Gates At the center of this will be the idea of digital convergence. That is, taking all the information-books, catalogs, shopping approaches, professional advice, art, movies - and taking those things in their digital form, ones and zeroes, and being able to provide them on demand on a device looking like a TV, a small device you carry around, or what the PC will evolve into [1]. But mobile devices still lack in resources as compared to conventionally used data processing devices such as pcs and laptops. So there is severe need of technology so anyone could access data anywhere as everyone is busy in job and moving concerned to work. So, the solution is Mobile Cloud Computing (MCC).

\section{MCC}

Mobile cloud computing aim at using cloud computing technique for storing and processing data outside the mobile device. Examples of MCC like email, twitter, face book, Google drive etc.. MCC in simplest terms can be referred as infrastructure where both data storage and data processing happens outside the mobile device.

Mobile cloud computing is an emerging cloud service model following the trend to extend the cloud to the edge of networks. It includes numerous mobile devices that are closely associated with their users. They will be directly involved in many cloud activities that extend the Cloud boundaries into the entire cyber physical system. [2]. In Mobile Cloud computing mobile users got all the services provided on cloud in his/her mobile device and can use those services same as on conventional devices such as PCs or laptops. MCC is merging of mobile network and cloud computing which make life even simpler for moving people. Mobile Cloud Computing is a technology that influences integrated resources of varied clouds and network technologies toward limitless mobility, functionality and storage. It serves a mass of mobile devices anywhere, anytime through different channels, being either Ethernet or Internet regardless of diverse environments and platforms [3]

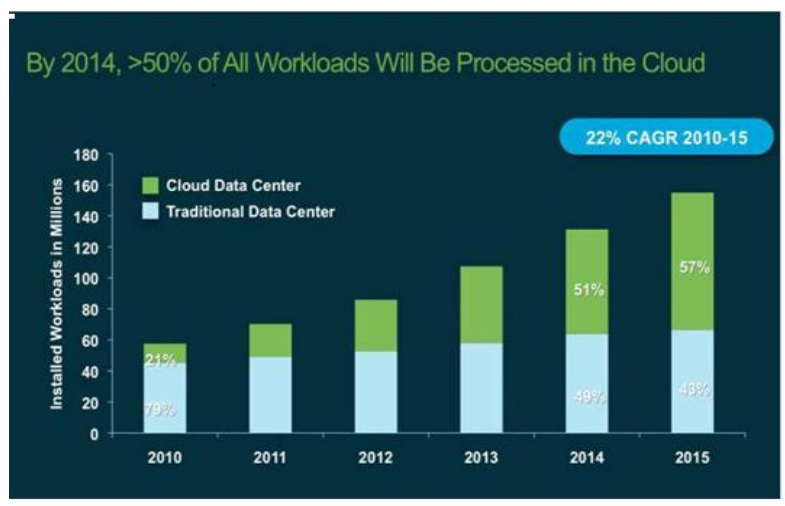

Figure1: Workload distribution

As the data shown in figure we can analyze the importance of cloud computing for mobile devices. Workloads through mobile data centers were $21 \%$ in 2010 rest were through traditional data centers, but in 2015 it will be $57 \%$ through mobile data centers and rest will be through traditional data centers.

\section{BACKGROUND}

Mobile cloud computing id inherited from cloud computing. Cloud computing is in trend from about 2009 when the users of cloud computing were only about $20 \%$ and up to 2015 the expected rate of mobile users is $70 \%$.

Cloud Computing refers to a technology where data or application software are saved online on servers, users can operate on data without installing any software. That means when ever user wants he can open browser and use files saved on internet. Most common example of cloud computing is Gmail. According to NIST

Cloud computing is a model for enabling ubiquitous, convenient, on-demand network access to a shared pool of configurable computing resources (e.g., networks, servers, storage, applications, and services) that can be rapidly provisioned and released with minimal management effort or service provider interaction. This cloud model promotes availability and is composed of five essential characteristics, three service models, and four deployment models [4]. The term "Cloud" is analogical to "Internet" word cloud is introduced in cloud computing based on cloud drawing used in past to represent telephone networks. Most of us are 
habitual of hitting "effect" without knowing the "cause". Today every sector business, working or government sector is based on computer application. Though not every user is capable of understanding the internal operations of computer, yet they get benefit from computer and most of them from internet. This is possible through cloud computing. According to Dinh et. al.[15] Cloud computing (CC) has been widely recognized as the next generation computing infrastructure. CC offers some advantages by allowing users to use infrastructure (e.g., servers, networks, and storages), platforms (e.g., middleware services and operating systems), and software's (e.g., application programs) provided by cloud providers (e.g., Google, Amazon and Sales force) at low cost .If we talk business related cloud computing is such type of cloud computing environment where business owner outsource their need of application services to third party and whenever they need to use the application like database, accounts, emails etc they access it from internet.

For example if any organization have small business, it will need small servers for database, application or account. Usually servers need very high speed for computations but for laptops, PCs have low computation power and much lower cost and more is to maintain client server environment. Organization will need much higher skilled workers. So to cut off this cost organization can use cloud computing architecture because according to cloud computing architecture they need not to install specific software or maintain servers. They just need to pay a fixed amount to the third party data center and it becomes the responsibility of the third party to manage data. Organization can access its data through internet browser. In overview we can say that all data is provided as a service. User can have access to these services without having the knowledge on how to manage resources and thus user can concentrate more on his core business without worrying about the resources to manage the business process. Cloud computing eliminate the use of buying additional hardware.

\section{CLOUD COMPUTING ARCHITECTURE}

\subsection{Deployment Model:}

There are four types of deployment models:

1.Public cloud: It is most popular type of model. In public cloud third party provides the space for data storage and computing power for all applications. Service providers make resources which are available to the whole public over internet. Amazon and Google apps is an example of public cloud computing.

2. Private Cloud: it is infrastructure operated only for single organization but it is managed by third party. All data center is to be set up and have to bear all installation and maintenance cost. But organization has complete control on data. In private cloud there is more security.

3. Community Cloud: It shares applications between some organizations which have common type of needs. Community cloud can be managed internally or by the third party.

4. Hybrid cloud: It comprises of two or more clouds. Clouds in hybrid remain unique but are linked together so Its easy to move data from one deployment model to another.

\subsection{Cloud Computing Service Architecture}

Cloud computing provide three type of services.

1. Software as a service (SaaS): Service

Provider will give user the capability of using service running on cloud infrastructure. This eliminates the need to install and run application consumer's computer. Applications are accessible from all client's devices using web browser .Consumer do not have to manage or control any network, server, data storage, OS etc

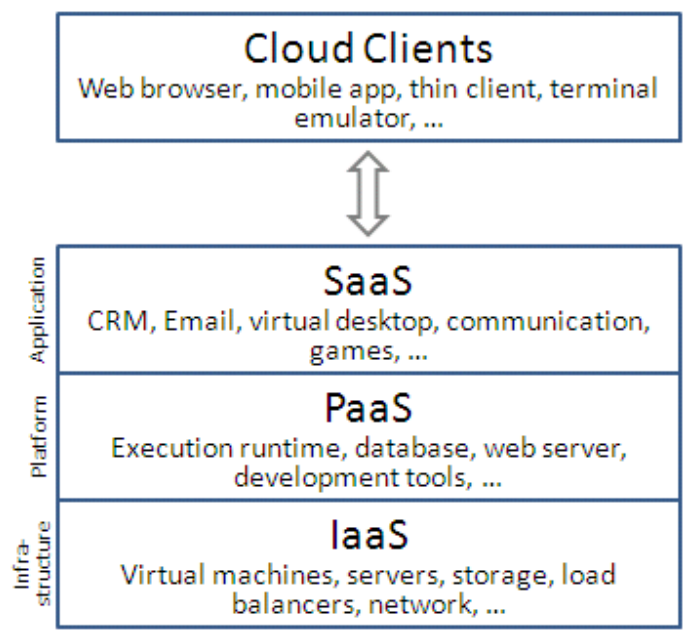

2. Platform as a service (PaaS): Platform as service facilitate deploying applications without cost and inconvenience of buying and managing the underlying hardware and software layers. In other words PaaS provides platform to develop their own application platform.

3. Infrastructure as a service(IaaS): Iaas provides the consumer the provision of processing, storage, networks and other resources. The service provider will bear the cost of server network, storage or backup etc. User have to pay and take computing service and build their own application software.

\subsection{Mobile cloud computing architecture}

We will discuss both conventional architecture and open mobster architecture. In openmobsster we will discuss services needed by two main Components: Front end and back end. Front end comprises of client's device and some more applications to run Cloud computing services. Back end is Cloud itself which encompass all related information stored in servers that client wishes to access. All these clouds in combination make cloud computing systems. This whole system is administered by central server that used to monitor client's demand and manage traffic of data flow. Software "Middleware" is used to let computers communicate that is connected to internet. Cloud system must have copy of all clients' data; in case of system breakdown cloud restore the data.

Architecture of MCC: 

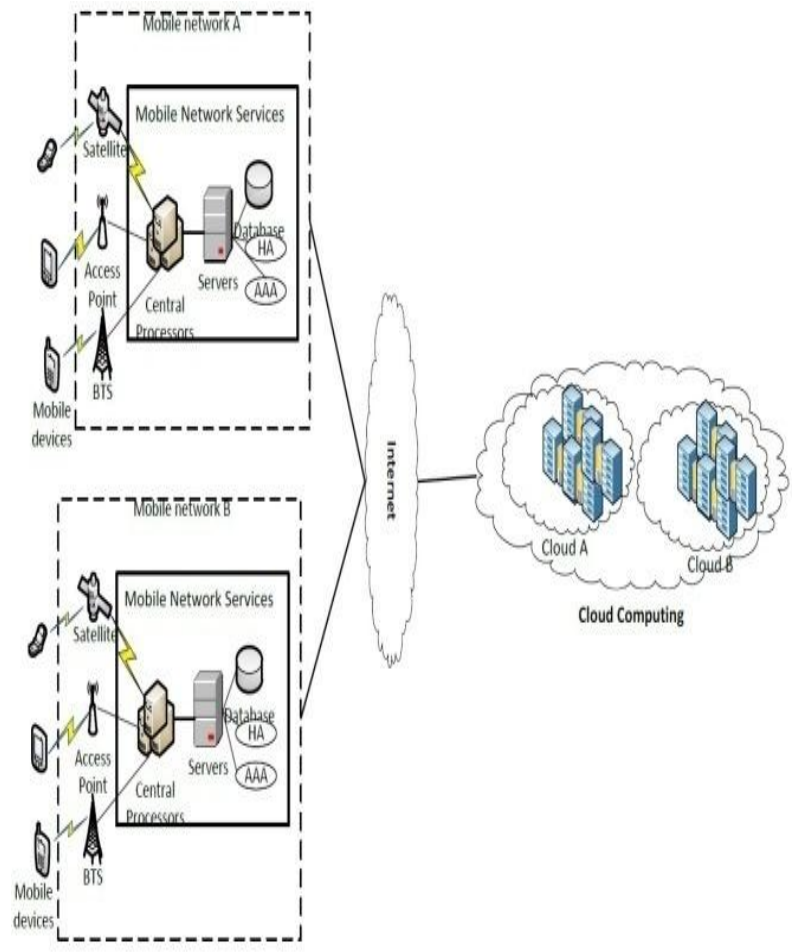

Mobile devices connect to network services through any satellite, access point or BTS (base transceiver station (BTS) which pass request to control serve connected to server providing services. Server provides services like AAA (Authentication, Authorization, and Accounting) based on HA. HA is a home agent that work as a router on a mobile devices' home network that maintains information about the device's current location. The home agent uses mechanisms to forward Internet traffic so that the device's IP address do not have to be change every time it connects from a different location. That mechanism is known as tunneling. Any data or request is submitted on database. This all processing is still on mobile network itself. Request by user is then passed to cloud through internet. Cloud controller process the request to provide required data to user.

\section{OPEN MOBSTER ARCHITECTURE}

Open Mobster [6] is an open source platform for developing Cloud connected Mobile Apps. It provides the following services[7].

\subsection{Features}

- Sync Platform: Automatic bidirectional data sync between the devices and the Cloud

- Push Notifications: A platform-agnostic Cloud-initiated Push Notification System

- Location Aware Apps: An framework for creating endto-end Location Aware Apps

- Mobile RPC (Remote Procedure Call): A simple name/value pair based method for invoking service components in the Cloud

- Management Console: A GWT/SmartGWT based App to administrate the system

\subsection{Mobile cloud services needed by client}

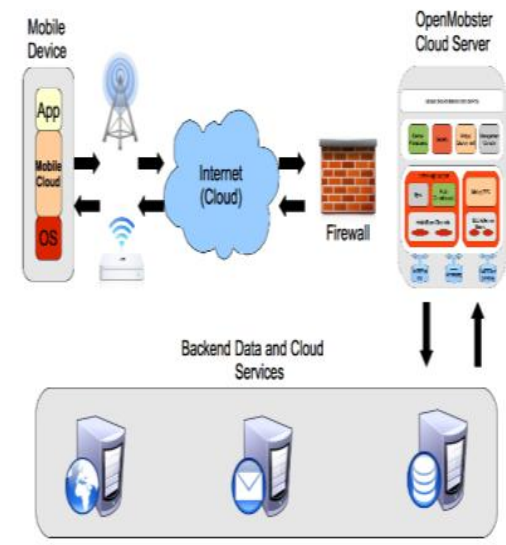

1. Sync: Sync is a service to synchronize data on to cloud. Any changes made to data or application will be synchronized to open up next time according to your settings.

2. Push: all the notifications being sent by cloud server are managed by push. Users need not check or update necessarily any notification.

3. Offline App: Offline App is platform to co ordinate between low level services . users need not to program for any synchronization. offline App automatically manage synchronization . users just need to click for sync.

4. Network: network is just a channel that establishes connection between mobile network and cloud server. User need not to know underlying functioning of network.

5. Mobile RP: Remote Procedure Call Simple Mobile RPC (Remote Procedure Call) Exposes your server-side coarse grained business services. These services are invoked via a simple RPC mechanism without any low-level programming like http-client code, client side REST library, etc on the part of the App developer. There is a RPC API that is used for making these calls.

6. Database: Database store data for Apps .Database provide safe concurrent access to apps.

\subsection{Server side services}

A Cloud Server is located in the 'Cloud'. The server provides mobile-oriented features like data synchronization, real-time push, and mobile RPC etc. Cloud server is intermediate between the mobile device and the actual cloud data services being provided to mobile devices. According to Chetan et. al . [5] following are the services that should be provided to mobile apps

1. Sync : All your app data is automatically managed by the on-device cloud stack. Any operations that have occurred in offline mode are auto-tracked. When the platform detects network connectivity, it auto-syncs the information back with the cloud. In the sync process, it brings in any new information that may have appeared on the cloud while you were disconnected. Server Sync service synchronizes device side App state changes with the backend services where the 
data actually originates. It also must provides a plug-in framework to mobilize the backend data.

2. Push : The Push mechanism uses a pure network/socket based approach instead of clunky out of band methodologies like sms alerts, text message, or email alerts. The Push notifications happen inside the app's environment Server Push service monitors data channels (from backend) for updates. The moment updates are detected, corresponding notifications are sent back to the device. If the device is out of coverage or disconnected for some reason, it waits in a queue, and delivers the push the moment the device connects back to the network.

3. Secure Socket-Based Data Service: Depending on the security requirements of the Apps this server side service must provide plain socket server or a SSL-based socket server or both.

4. Security: Security component provides authentication and authorization services to make sure mobile devices connecting to the Cloud Server are in fact allowed to access the system. Every device must be first securely

provisioned with the system before it can be used. After the device is registered, it is challenged for proper credentials when the device itself needs to be activated. Once the device is activated, all Cloud requests are properly authenticated/authorized going

5. Management Console: Every instance of a Cloud Server must have a Command Line application.

\section{ADVANTAGES OF MCC}

1. How battery lifetime can be extended by MCC:

Battery is main component of mobile devices. structure of battery need a change to co- operate with present applications. Secondly, application cost for mobile users is also issue of concern. So, MCC is solution to problem. The solution is offloading technique in MCC.

Offloading: computation offloading technique transfer large computations and complex processing from device which have limited resources like mobile devices to machines which have full resources that is cloud server in cloud . this will result in less power consumption because offloading technique will avoid long application execution time on mobile device.

\section{Storage capacity:}

Challenge in storage capacity is today user need more and more capacity on mobile devices to save information that is essential or for his/her entertainment sake . but as the storage capacity increase weight of device also increases. Then what could be the solution to save large amount of data on low weight devices?

Again the solution is MCC.

MCC is developed to enable mobile users to store the large amount of data on cloud through network server. Some best examples of such kind of service is Flicker, Google Derive, Amazon S3, Gmail etc.

\section{Reliability:}

Of course every user needs a reliable backup for their data. MCC provide a reliable source to store data that is cloud. User need not to worry about loss of data on crash of device or loss of device.

Storing data or running applications on cloud is very safe way to improve reliability since data is automatically backed up on cloud.

\section{Dynamic provisioning:}

Dynamic on-demand provisioning of resources on a finegrained, self-service basis No need for advanced reservation. A dynamic service provisioning, i.e. dynamic resource allocation and dynamic deployment of services in a distributed environment enables high flexibility and optimized usage of the infrastructure.[9] Cloud provisioning is the allocation of a cloud provider's resources to a customer. [16] When a cloud provider accepts a request from a customer, it must create the appropriate number of virtual machines (VMs) and allocate resources to support them. The process is conducted in several different ways: advance provisioning, dynamic provisioning and user self-provisioning. In this context, the term provisioning simply means "to provide." With advance provisioning, the customer contracts with the provider for services and the provider prepares the appropriate resources in advance of start of service. The customer is charged a flat fee or is billed on a monthly basis.

With dynamic provisioning, the provider allocates more resources as they are needed and removes them when they are not. The customer is billed on a pay-per-use basis. When dynamic provisioning is used to create a hybrid cloud, it is sometimes referred to as cloud bursting.

With user self-provisioning (also known as cloud selfservice), the customer purchases resources from the cloud provider through a web form, creating a customer account and paying for resources with a credit card. The provider's resources are available for customer use within hours, if not minutes.

5. Scalability:

Mobile applications can be performed and scaled to meet the unpredictable user demands

Service providers can easily add and expand a service

\section{APPLICATIONS OF MCC}

\section{Mobile commerce:}

Mobile commerce usually known as M-commerce is a model for commerce on mobile devices. Mobile Commerce, or mCommerce, is about the explosion of applications and services that are becoming accessible from Internet-enabled mobile devices. It involves new technologies, services and business models.[8].Some categories of mobile commerce

As the numerous products and applications are provided through m-commerce, but there are various challenges associated with m-commerce like low network bandwidth availability, high complexity in mobile device configurations, and the major issue is of security/privacy. As a result, mcommerce applications are used through computing environment to solve these issues. But PKI (public key infrastructure) can be solution to this problem. PKI mechanism uses an encryption-based access to ensure users private and secure access to the cloud stored data.

\section{Mobile Learning:}

Mobile learning techniques is rapidly adopted by youth. Mlearning is combination of e-learning and mobility. Any data can be downloaded on mobile while busy in work. As we see, traditional e-learning has limitation of slow transmission rate, resources that are used for traditional learning are limited in number, also the devices which are used for traditional $\mathrm{m}$ learning are high cost, so, the solution to theses traditional elearning problem is cloud based learning. Through $\mathrm{m}$ learning remote resources can be accessed.

3. Mobile Healthcare: 
In traditional medical treatment small storage, medical errors are some problems that occur to users. Through M-healthcare mobile users can convientelly access resources whenever user is in need of healthcare. Many of healthcare services are provided on clouds which are on-demand. The advantages of healthcare to access healthcare information and manage expenses on health. Varshney et. al. [10] discussed some examples and devices related to healthcare

1. Some comprehensive healthcare services are provided to users which help to monitor users ate anywhere anytime through mobile device.

2. Intelligent emergency management system manages and co-ordinate the fleet of emergency vehicles effectively and in time when receiving calls of accident.

3. Health aware mobile devices detect blood pressure, pulse rates and level of alcohol to alert healthcare emergency systems.

4. Pervasive access to healthcare information allows patients or healthcare providers to access the current and post information.

5. Healthcare expenses and other related charges can be managed through pervasive lifestyle incentive management.

\section{Mobile Gaming:}

As with advanced deployment of wireless network, new possibilities are opened to play internet games developed for pc's on wireless mobile devices.

According to developers of Mobile System Design Lab[11] may be due to constraint on hardware, memory and graphics processing, goal might be difficult to achieve using current client server architecture.

But this is also the fact that mobile gaming is main source of generating, revues for service providers. Mobile games need to be offloading to save energy. According to wang et. al. [12] through offloading devices can save energy for mobile devices so the time for mobile game playing increases. According to Karnik et.al. offloading is a solution to augment these mobile system capabilities by migrating computations to more resourceful computers. For better solution on energy saving cuervo et. al. [14] proposes a MAUI (Mobile Assistance Using Infrastructure), MAUI system helps in fine frained energy aware offloading of mobile code to cloud.MAUI divides application code at runtime based on communication network and processor to maximize energy savings.

\section{Melog:}

It is MCC applications that enable mobile users to share real time experience. The mobile users are supported by several cloud services such as guiding a trip, slowing images and videos and showing maps etc.

Video based mobile locations search capture a short video clip of surrounding buildings. The information is used in matching algorithm run on cloud to search for location of these buildings.

One hour translation is a translation service in which you choose language such as English to French , upload material and select expertise. It help mobile users to receive information translated in their language through mobile device.

A cloud become effective tool when mobile users require searching services through keyboard, tag based searching etc. with the help of certain social sites such as twitter and face book to search photos.

\section{SOME OF THE ISSUES}

Though mobile cloud make the application accessing and storing data easier but still there some issues that need to solved like low bandwidth. Wireless radio transmission sources are scarce as compared to wired transmission. This is hazard in application service because downloading and uploading is severally effected with speed of transmission. A solution was proposed by Jin et. al.[17] that users which are located in same area and are accessing same type of content should share bandwidth. But this will be possible only if users are involved in same type of content. Availability and heterogeneity are also the major issues need to be solved out. Availability is limitation of resources or inability to connect to server due to traffic congestion or low bandwidth. Heterogeneity problem occur when mobile devices interact through different radio access technologies. These were the issues on server side. Some computing side issues could be offloading in static and dynamic environment, in static offloading can consume more energy to process a short codes compared to local processing. In dynamic problem of offloading data ca not reach to destination or data can be lost while returning to user[15]. Second issue that can be discussed can be security issue. Privacy of data is main issue while using LBS(location based services) to provide private data on cloud and integration of data while using application is necessary issue to be handled these are just some of issues many more issues need concern like data access, quality of service etc.

\section{CONCLUSIONS}

From the above discussion we can conclude that mobile computing help user to save data on cloud easily and accessing service while moving make their job easy. But some aspects still need to be taken care of like low bandwidth is the major issue on mobile cloud, accessing data; security etc. issues should be solved. If these problems are solved then MCC will be a new revolution and can diminish the use of pc.

\section{REFERENCES}

[1] Bill Gates,"Information at Your Fingertips-2005," (COMDEX keynote speech, 1994)

[2] Dijiang Huang,"Mobile Cloud Computing", IEEE COMSOC MMTC E-Letter ,http://www.comsoc.org/ mmc/ 1/4 Vol., No., 2011

[3] Iraky Khalifa, Hala El-sayed and Islam Abd El-gaber, "Article: Enhanced Mobile Cloud Computing Platform", International Journal of Computer Applications 63(7):12-18, February 2013. Published by Foundation of Computer Science, New York, USA. BibTeX

[4] National Institute of Standards and Technology,www.nist.gov.inSpecial Publication 500-291

[5] Chetan, "cmw.pdf'

[6] Openmobster, www.openmobster.com

[7] Sohil Shah, Chief Engineer, Open Mobster ,http://www.quora.com/What-is-OpenMobster

[8] mobile commerce lab, http://mcom.cs.cmu.edu/

[9] Katharina Görlach and Frank Leymann University of Stuttgart, Germany," Dynamic Service Provisioning for the Cloud", Institute of Architecture of Application 
Systems, University of Stuttgart Germany, iaas.unistuttgart.de

[10] varshney $\mathrm{u}$, "Pervasive healthcare and wireless health monitoring " , Journal on Mobile Networks and ApplicaTIONS, 2007, 12(2-3):113-127

[11] Mobile system design lab, university of California, san Diego

[12] Li Z, Wang C, Xu R.," Computation offloading to save energy on handheld devices: a partition scheme" , In Proceedings of the 2001 international conference on Compilers, architecture, and synthesis for embedded systems (CASES), 2001; 238-246.

[13] Karnik Kumar, Jibang, Yung Hsiang Lu, Bhargava,"A survey of computation offloading for mobile systems", Mobile network Applications, DOI 10.1007/s11036-0120368-0
[14] Cuervo E, Balasubramanian A, Dae-ki C , "MAUI: making smartphones last longer with code offload", In Proceedings of the 8th International Conference on Mobile systems, applications, and services, 2010; 49-62.

[15] Hoang T. Dinh, Chonho Lee, Dusit Niyato and Ping Wang, "A survey of mobile cloud computing: architecture, applications, and approaches", Published online in Wiley Online Library (wileyonlinelibrary.com). DOI: $10.1002 / \mathrm{wcm} .1203$.

[16] http://searchcloudprovider.techtarget.com/definition/clou d-provisioning

[17] Kelenyi I, Nurminen JK, “CloudTorrent- energyefficient bittorrent content sharing for mobile devices via cloud services", In Proceedings of the 7th IEEE on Consumer Communications and Networking Conference (CCNC), $2010 ; 1$ 